\title{
Bridge Launching Construction Visualized in a Virtual Environment
}

\author{
Octávio P. Martins and Alcínia Z. Sampaio
}

Department of Civil Engineering and Architecture, Technical University of Lisbon, Lisbon, Portugal

\begin{abstract}
Virtual Reality techniques were used to develop an interactive application concerning the incremental launching construction of bridges, in the area of Civil Engineering. It was developed in order to facilitate understanding of the various phases involved in the construction. The visualization of the distinct physical steps of the construction is shown in the virtual application. The model makes it possible to view the physical evolution of the work, to follow the planned construction sequence and to visualize details of the form of every component of the work. It also supports the study of the type and method of operation of the equipment necessary for this construction activity. The application of visual simulation was designed to allow direct access to any stage of the constructive process in which it is based and may be viewed from any point within the virtual scene, thus facilitating their understanding. The access to the application can be established through a web page placed on the Internet. The model brings new perspectives in the training activity as a support to expose innovative methods or complex sequence construction.
\end{abstract}

Index Terms-Bridge construction, Interaction, Visual Simulation, Virtual Reality.

\section{INTRODUCTION}

The aim of the practical application of the virtual reality (VR) model, is to provide support in Civil Engineering training namely in those areas relating to bridges and construction process. The model can be manipulated in classroom-based education, or in distance learning based on e-learning technology or by professional related with Civil Engineering.

The human perceptual and cognitive capabilities were taken into account when designing this visualization tool. Further more, associated with each new component or step there are integrated information concerning the construction activity, allowing the consult of the require data in any phase. The virtual three-dimensional (3D) models present a menu concerning the development of the activity linked to each step, so the trainers and the learners can interact with it when explaining the method or studying it.

In order to create models, which could visually simulate the progressive sequence of the process and allowing interact with it, techniques of virtual reality were used. When modeling 3D environments a clear intention of what to show must be planned, because the objects to display and the details of each one must be appropriated to the goal the teacher or designer want to achieve with the model. In addition, the use of techniques of virtual reality on the development of these didactic applications is helpful to education improving the efficiency of the models in the way it allows the interactivity with the simulated environment of each activity. The virtual model can be manipulated interactively allowing the teacher or student to monitor the physical evolution of the work and the construction activities inherent in its progression. This type of model allows the participant to interact in an intuitive manner with the three-dimensional space, to repeat the sequence or task until the desired level of proficiency or skill has been achieved and to perform in a safe environment. Therefore, this new concept of VR technology applied to training models brings new perspectives to the teaching of subjects in Civil Engineering training [1].

The developed application makes it possible to show the physical evolution of the works, the monitoring of the planned construction sequence, and the visualization of details of the form of every component of the construction. It also assists the study of the type and method of operation of the equipment necessary for this construction methodology. Specialist in bridge design were consulted and implicated in the execution of the educational model in order to obtain an efficient and accurate didactic application. The pedagogic aspect and the technical knowledge are presented on the selection of the quantity and type of elements to show in the virtual model, on the sequence of exhibition to follow, on the relationship established between the components of the construction, on the degree of geometric details needed to present and on the technical information that must go with each constructive step. From this application it is possible to obtain 3D models corresponding to different states of their shape, simulating distinct stages of the carrying out process [2].

The present model attends the incremental launching method of bridge deck construction. This application follows another VR models developed within the Technical University of Lisbon at the Department of Civil Engineering, concerning construction works (Fig. 1): one model shows the execution of an external wall, a basic component of a building; another model presents the cantilever process of bridge construction; and the third model presents the construction of a roof of a building [3]. 


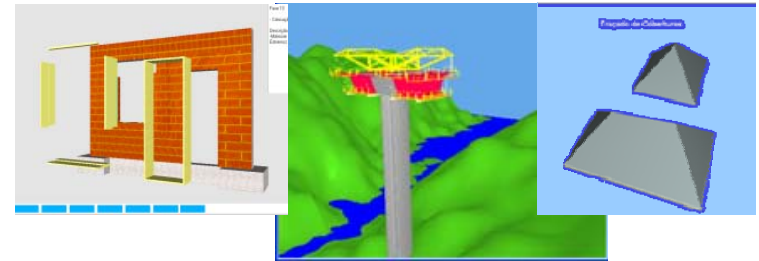

Fig. 1. Didactic virtual models in construction.

To facilitate understanding of the various phases involved in the incremental launching usage method, it was made an application of visual simulation through programming and 3D modeling in virtual reality environment of the elements involved in construction. In this text, the main aspects of the constructive process by incremental launching are mentioned, the assumptions of the case study to use are defined, and with the support of tools for programming in the virtual environment the construction of the virtual deck is planned.

\section{BRIDGE CONSTRUCTION METHODS}

The construction method for the execution of bridges and viaducts must be taken into account from the earliest stages of the design [4]. Due to its importance, and because it involves several technical teams that depend on it, his detailed knowledge is very important. Sometimes this understanding is complicated by increasing innovation and upgrading of new construction methods of the main construction companies that compete with each other.

The processes of construction have a huge influence on the selection of the cross section of the deck and, consecutively, the structural solution. This solution must verify the principal objectives, inherent in any structure: functionality, security, durability, economics and aesthetics.

Perhaps in no other works in the field of engineering structures, the constructive process affects the design as the bridges. The main methods of construction most often used are the false work supported on the ground, the formwork carriage and cantilevering method made by formwork travelers and the construction by incremental launching. Fig. 2 illustrates these methods:

- The false work supported on the ground, with scaffolding, is the most traditional method, due to its easy implementation and economy, and is the oldest construction type of bridges. It is appropriate to viaducts with low height (up to $20 \mathrm{~m}$ ), and always supported on a firm area. The false work does not restrict the type of section, therefore the deck may be supported by ribs, beams or with box girder cross section [5];

- The formwork carriage emerged with the need for extensive construction of viaducts that could run freely without any limitation by the type of terrain or the height of structure. The formwork can be used to support in-situ concrete, or just to elevate and suspend precast staves. It is suitable for the execution of long bridges, straight or with a small curvature. The span lengths must be equivalent, with values of 30 to $60 \mathrm{~m}$ and can reach $70 \mathrm{~m}$. The deck is generally supported by beams or ribs. The basis of this operation method is to support the formwork on the final elements (pillars and abutments), may also use part of the built deck like support too. Thus, the displacement of the formwork is done from pier to pier [5];

- The bridges built by the cantilever method have its structural model strongly conditioned by the constructive process. The structure advances from a short deck segment on top of a pier symmetrically in segments of about $3 m$ to $5 \mathrm{~m}$ length to the mid span or to an abutment. This system is the most used for building bridges with spans of $60 \mathrm{~m}$ above, regardless of height. It is also used with some frequency in the construction of arches of bridges and has a considerable field of application in the bridges built by precast staves. For extensions of less than $50 \mathrm{~m}$ range, can be equated with the earlier solutions, depending on the number of piers and height [6].

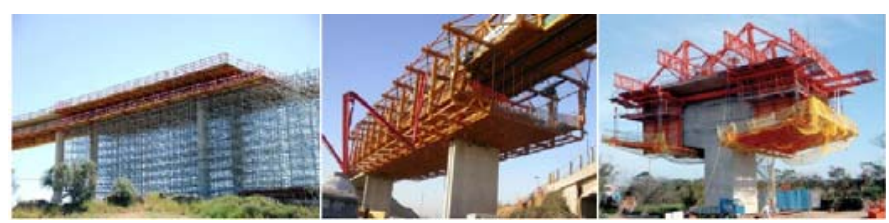

Fig. 2. Bridge construction methods.

\section{INCREMENTAL LUNCHING METHOD}

The incremental launching method consists of casting $15 \mathrm{~m}$ to $30 \mathrm{~m}$ long segments of the bridge superstructure in a stationary formwork to push a completed segment forward with hydraulic jacks along the bridge axis. In 1961-63 the first bridge of reinforced concrete was built through incremental launching [7] in the city of Guyana, Venezuela. Andrä strengthened this constructive process, in 1965, with the discovery of the advantages of application of Teflon on the support, allowing greater slip of the deck during its incremental progress.

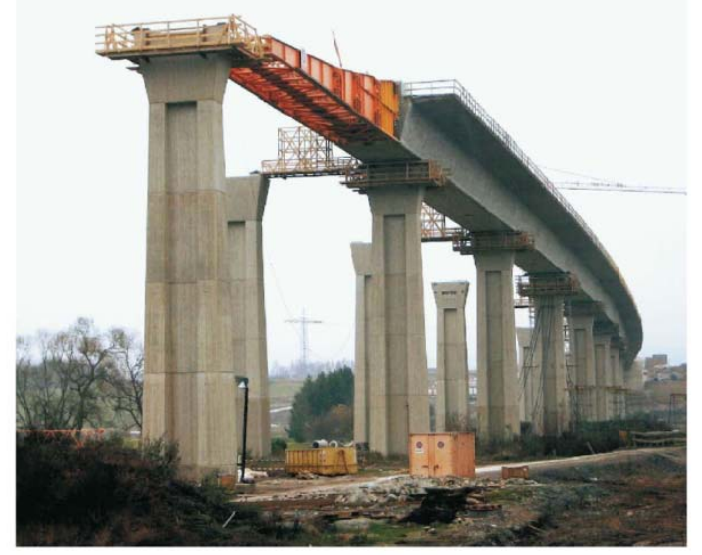

Fig. 3. The construction of a bridge decks using the method on incremental launching (http://www.abdnb.bayern.de/).

The incremental launching is designed to be applied in viaducts over valleys and mountains with spans of about $50 \mathrm{~m}$ [8]. However, its use is common in flat areas where access at ground level may be conditioned, or on which it is 
inappropriate to impose disturbances, such as works on traffic, railways, etc.

The length of the optimal span is between 45 and $50 \mathrm{~m}$. The method can be applied also in bridges of reinforced concrete and in steel bridges and mixed. The bridge axis can be straight or curved circular. It is estimated that the minimum length of a bridge or viaduct to justify the cost of its implementation is $200 \mathrm{~m}$ [7]. The application of this constructive process requires that the height of the cross section needs to be constant over its entire length, because each section will have different states of bending moment and thus different tensions. The appropriate type of section is the box girder, as they have a good relationship between the top and bottom flexural modulus. The longitudinal pre-stressing of the deck is divided into 2 groups: the pre-stressing of constructive stage, installed and placed under tension before the deck launch, and the extra pre-stressing, placed under tension after the deck reach its final position.

Specific equipment for the proper use of the incremental launching method is needed: the launching equipment, responsible for the advancement of the deck; the support bearings, which facilitates the sliding of the deck on the piers; the launching nose, located in front of the deck. Through the application of visual simulation that was developed in this work, it is possible to observe the geometry and operation of equipment in the process. The developed VR model provides a contribution to the dissemination of information concerning this construction method, through a recording of visual simulation of the phases and the equipment that comprises the construction process.

\section{CASE STUDY}

The type of bridge that was used for this work resulted is a compilation of several cases reported in the literature and various photographic records available in some construction companies and engineering schools such as Federal Polytechnic School of Laudanize (http://is-beton.epfl.ch).

The cross section of the deck, abutments, geometry and size of the piers are based on the Itztalbrücke viaduct, built in 2007, near Rodental, Germany (www.abdnb.bayern.de, 2008). As the constructive process involved is due to a combination of cyclical actions, the case study consists only in one deck, supported by 6 piers, showing however the same central span with $58 m$ (Fig. 4), like the viaduct Itztalbrücke.

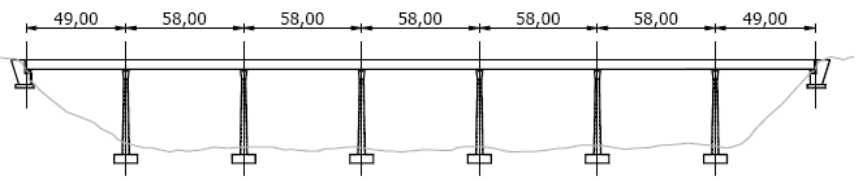

Fig. 4. Longitudinal view of the case study bridge.

Given its size, this virtual bridge is included in the group of long bridges and therefore the launching equipment could be the system Eberspächer [www.eberspaecher.org, 2008]. This equipment was referenced explicitly by Gohla [8] as owner of all the capabilities for this function. The bearing supports, used in construction phase, does not have any commercial reference because it was designed from the specifications and dimensions specified in [7]. The piers and abutments are fully built in visual model, because its implementation in work does not depend on the constructive process of the deck.

\subsection{Geometric Modeling}

Every elements needed in the virtual scenario were modeled and then the interaction was programmed using the some software based on the virtual reality technology.

For the creation and modeling of the elements was used AutoCAD, from AutoDesk. For the definition of the virtual interaction the EON StudioTM [9], from EON Reality was used. The software Eon StudioTM is a tool for creating interactive applications in 3D environment. The TU Lisbon has their licenses for their use, which are available for research in a laboratory. To establish the communication between EON StudioTM and AutoCAD was used 3ds Max, also from AutoDesk.

The unit of length used was the meter. The origin of the reference was defined at the base of the casting yard, represented in Fig. 5. Thus symmetry is obtained by the longitudinal vertical plane and the segments will be advanced from the vertical transverse plane $(y=0)$.

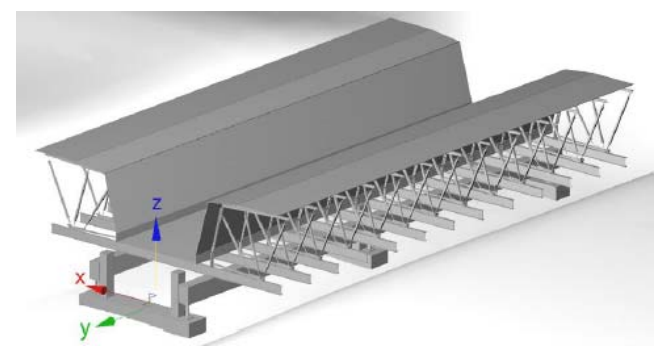

Fig. 5. Reference adopted for the modeling and programming of objects.

The form used for the 3D modeling was, in most cases, make the piece in two dimensions (2D) and then extend that section to create a solid. Later, using rotations, unions and subtractions between solids, inherent properties of AutoCAD, obtained the desired element. The object that will move during the simulation were visually differentiated by assigning the "layers" or color in AutoCAD in order to be identified by the transition software (3ds Max). Taking as example the false work used in the construction of segments of the deck, the Fig. 6 shows one of its elements. Through the operations described above, the metal parts were designed, then copied along the longitudinal direction of the deck and mirrored according to the longitudinal vertical plane (with $\mathrm{x}=0$ ).

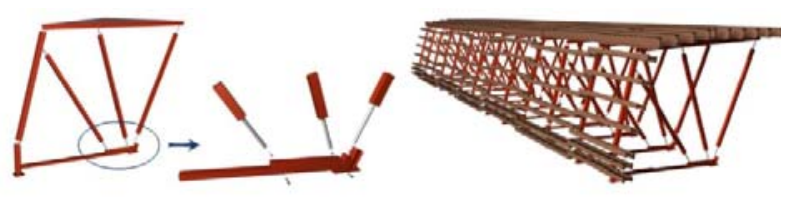

Fig. 6. Exterior formwork of the casting yard. 
Finally, the wood beams were created, to make the connection between the score and shuttering, and, with the same procedures, were placed in their correct position, as shown in the third image of Fig. 6.

The remaining objects were executed in the same way, varying the geometry. It was necessary to carefully assess what level of detail required for modeling in view of its presentation in virtual reality, because the geometric level of detail will be set depending on the purpose with which the interactive model is created.

For the representation of reinforcement, shown in Fig. 7, one steel mesh was designed in two dimensions. In 3ds Max these lines were setup as a way to go for a section in order to form a solid at each bar. It was not necessary to model all the reinforcement of the bridge, but only for a segment. The cross section used for steel bars was the rectangular instead the circle, to minimize the number of vertices and faces of all.

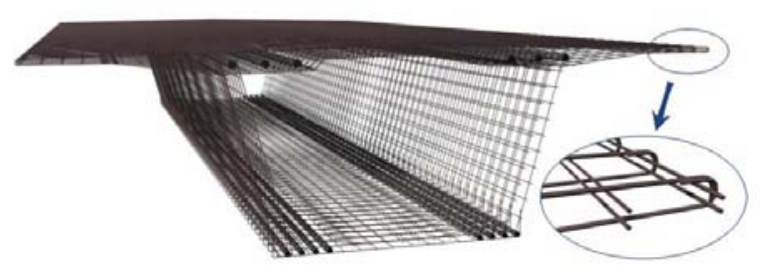

Fig. 7. Reinforcement steel mesh.

The launching nose was the most complex object produced in this work. His assembly in place is very repetitive, and will be showed in the application virtual, but the geometry of its elements is very uneven, as shown in Fig. 8. The application of materials in 3ds Max is in itself a matter of high complexity. It is possible to reproduce material very close to reality due to the large number of tools and options that the software provides. The materials created in 3ds Max, in general, could have been made directly in the EON Studio. The 3ds Max was chosen because it has a library with some pre-defined materials, and has a working platform frankly better. It is possible to change the characteristics of a material, such as color, brightness, roughness, and other, and instantly see the effects.

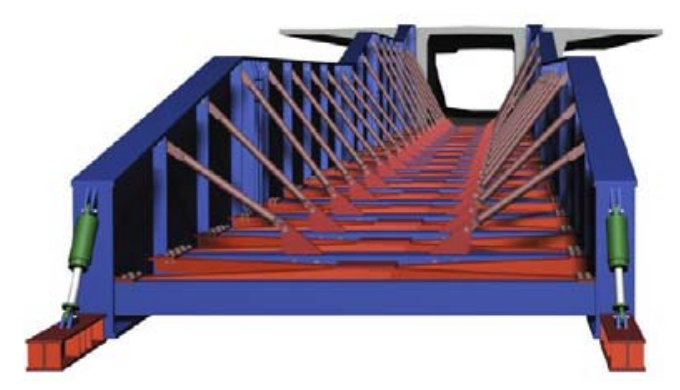

Fig. 8. Launching nose.( Color Plate 7)

For this work were essentially created two types of materials, with the use of image mapping as their difference. Through mapping coordinates and other properties, it is possible that the image is repeated in different directions.

The Fig. 9 shows the abutment of the bridge as one of the objects where the texture used has a concrete image. Besides the image applied in the concrete elements, was used an image of different texture to represent other material, wood, to be applied to the shuttering plates and the beams used for temporary support of the launching nose, represented also in Fig. 9.

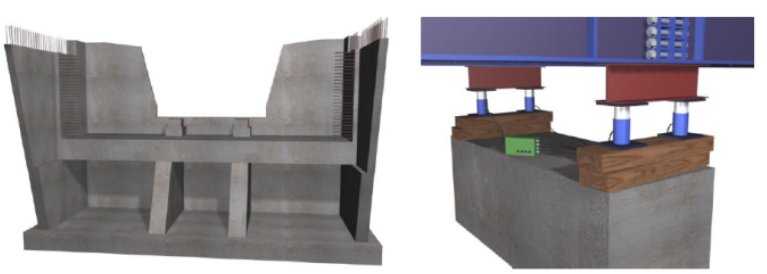

Fig. 9. Abutment and temporary support for the launching nose.

The remaining objects, mostly because they are the metallic type, can be set only with a material based on color, brightness, reflections, roughness, etc. Noting the hydraulic equipment, represented in Fig. 9, it is possible to check the differentiation between two materials, in addition to color. The outer cylinder, blue, has a uniform tone. The inner cylinder, light-colored, has a gloss and the reflection inherent of a sliding surface.

\subsection{Programming of interactive model}

In this item will not be exhaustively described all the procedures used to perform the programming and design of the application. Once all objects created and arranged in the correct position on the reference chosen, they were exported to the program EON StudioTM in order to start the programming of the virtual model.

The Fig. 10 displays the desktop of the EON StudioTM. In the middle window is available the hierarchical tree of the elements involved in the application to create. The hierarchy in this system facilitates the highly oriented programming with objects. During programming the nodes are added, which are essential for the design and implementation. By selecting any node in the central window, its properties can be viewed and edited in the window on the right. The map of the simulation is in the left window. In this board, and quite intuitive, there is established the communication between the nodes, forming a network of connections.

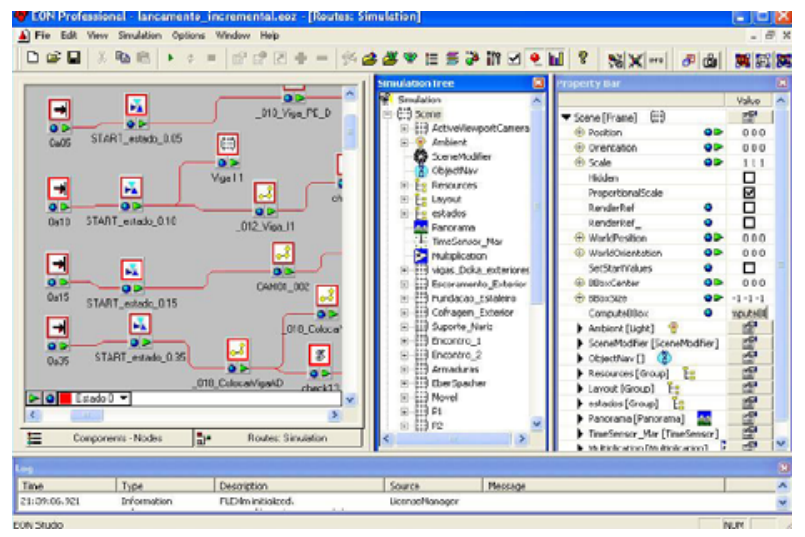

Fig. 10. Interface of EON StudioTM 
With the purpose to give some immersive capacity to the application, were used two nodes from the EON system. The first node, of type "cgMaterial," served to create the river by the mixing of colors and the definition of other parameters. The second node, of "panorama" type, served to create the virtual environment of the scenario. This node allows the definition of an image for the sky, horizon and floor. The user will have freedom to move freely in the virtual environment, so the images set to the horizon and sky, must grant the projection to 360 degrees. To achieve the effect of movement of the river, the nodes of type "timesensor", "multiplication" and "cgMaterial", were dragged to the map of simulation, with the names "timesensor_Mar", "Multiplication" and "Water", respectively, as represented in Fig. 11. The first node will be active during the application and is responsible for sending impulses to the node "cgMaterial (water)", after amplified by a multiplicative factor. These pulses provide a constant change of the parameter "time" of the node type "cgMaterial (water)", in other words, to change the amplitude of the waves in this material, thus creating the illusion of their movement.

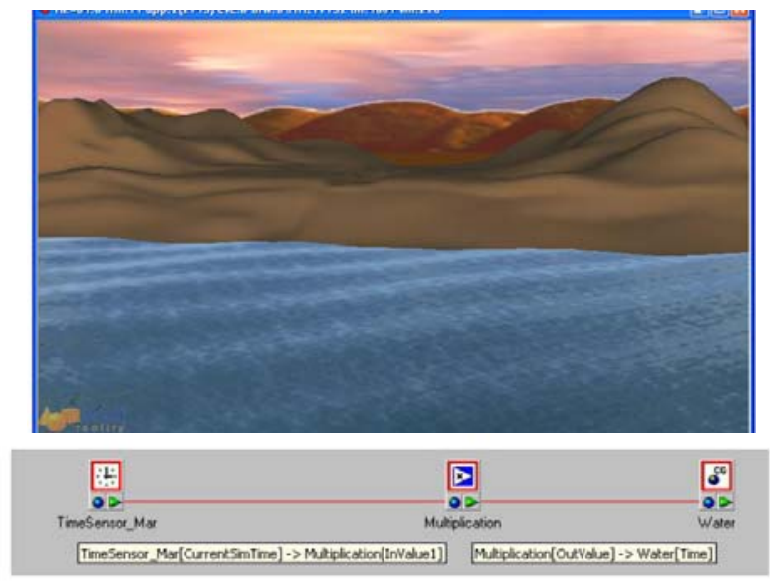

Fig. 11. Simulation of the movement of the river.( Color Plate 8)

After defining the necessary considerations and details on the framework of virtual scenario, the planning of the sequence of events to create was established.

\subsection{The Construction Sequence}

In order to report an overview of the construction place the camera points initially to the casting yard. At this stage just the abutments, piers and beams of the foundation of the yard are visible (Fig. 15).
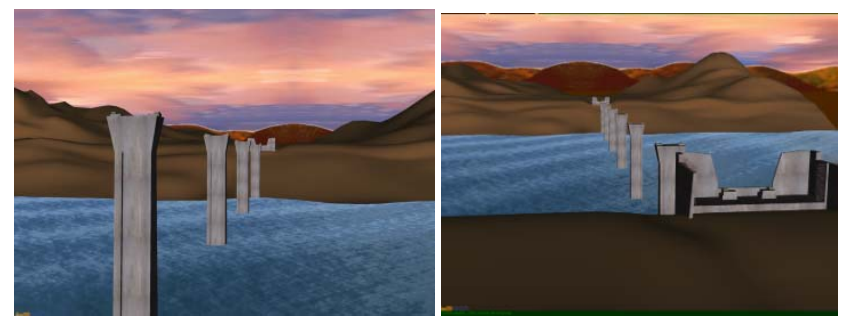

Fig. 12. Visualization of the abutments, piers and the casting yard.
Next, the exterior form work composed of 26 identical elements is building up. So, only the assembly of one element is visualized in detail. During the animation, the position of the camera and its movement are synchronized to show the details of the elements or the assembly type and also an overview of the working place (Fig.13)
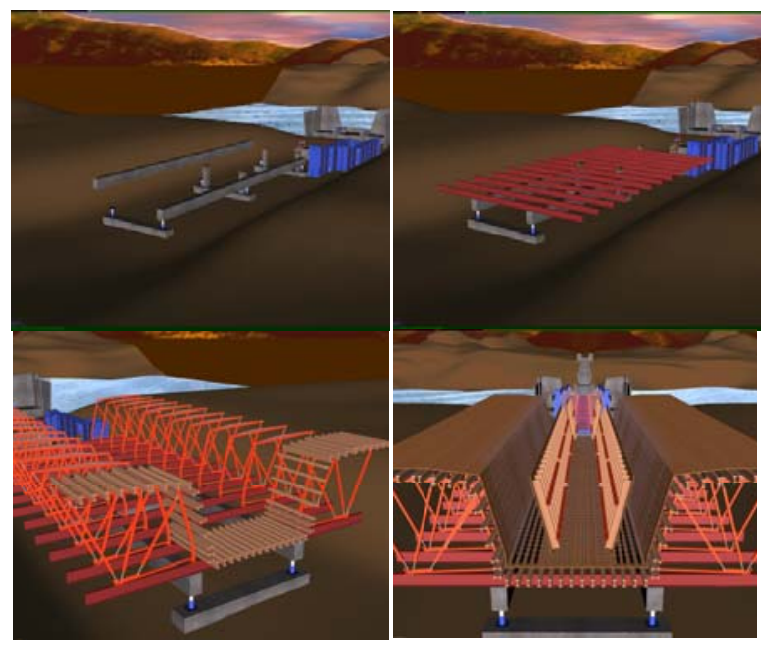

Fig. 13. Visualization of the exterior formwork structure.

After placing the external panels of the shuttering and the reinforcement mesh, starts the visual simulation of the casting work. The elements that make up the interior false work are placed incrementally, starting with the metallic support, followed by the longitudinal beams and finishing with the implementation of shuttering panels (Fig. 15).
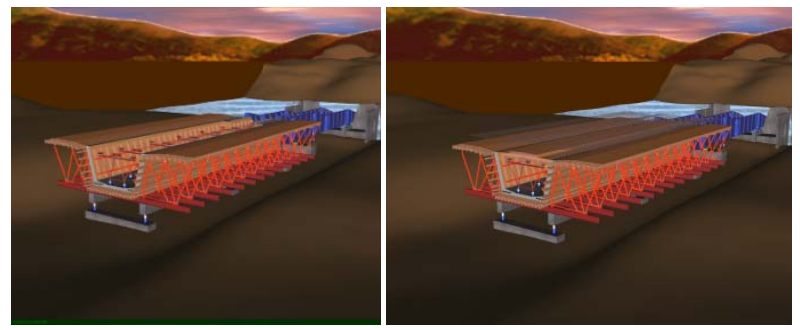

Fig. 14. Visual simulation of the casting work of the first deck segment.

Next, the assembly of the launching nose is installed. The camera is adjusted to allow the correct visualization of this work. After casting the first segment the displacement of this element takes place. For that the temporary support of the nose is removed and the segment is separated from the shuttering (Fig. 15).
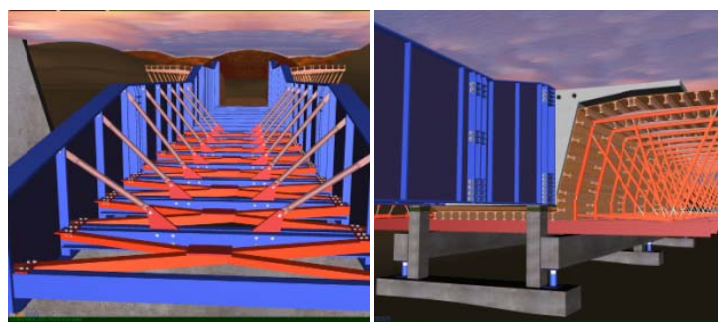

Fig. 15. Visualization of the metallic launching nose. 
To represent the advance of the deck, the launching equipment appears in detail, and it consists of two parts located under each web of deck, and thought four movements illustrated in Fig. 16, provides the launching of the segments. This cycle is repeated until the progress of the first segment reaches about $31 \mathrm{~m}$.

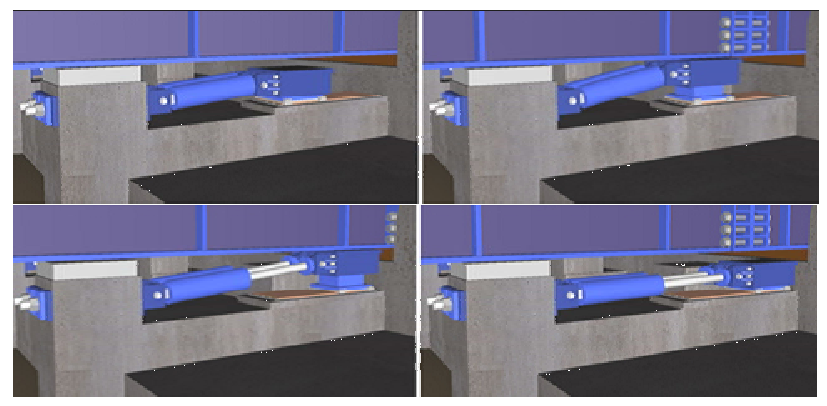

Fig. 16. Displacement of the launching nose.

The arrival of the nose to the first pier is achieved during the advance of the second segment. Fig. 17 illustrates the transposition process of the pier by the nose. In it the small brown parallelepiped are the launch pads and are placed manually by workers between the nose and the temporary support placed over each pier. The camera is positioned to show properly the work. This phase of the animation is programmed to run various actions at the same time, adequately synchronized (details in reference [2]).
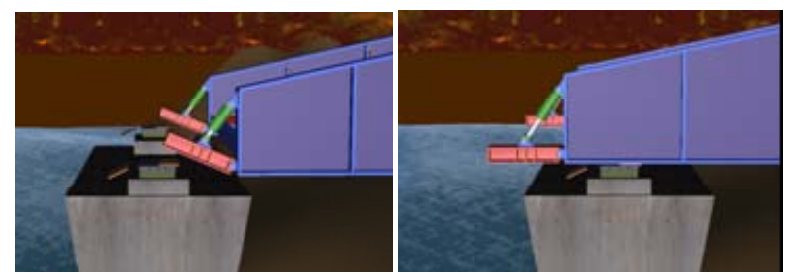

Fig. 17. Nose arriving at a pier.

The construction of the remaining segments is performed in fast mode, because the process is identical to the initial segment (Fig. 18).
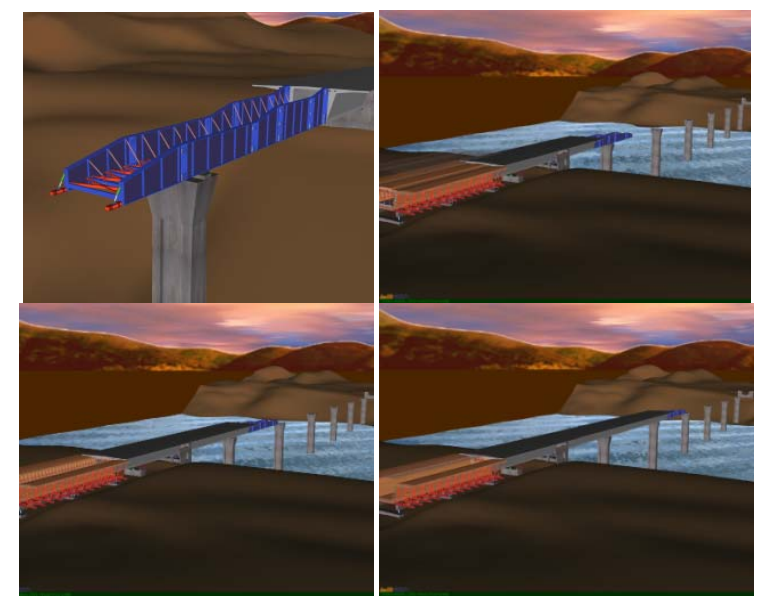

Fig. 18. Sequence of the incremental launched of the deck.
Already in the final phase of construction, the nose arrives at the abutment and the temporary support is replace by the definitive one (Fig. 19). The yard is removed and the space is covered of land (Fig. 20).

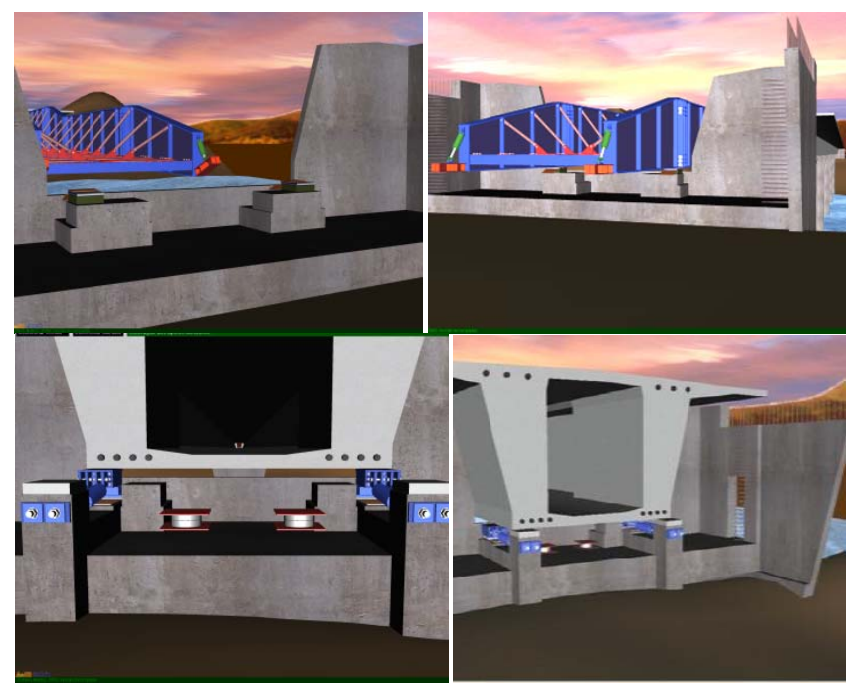

Fig. 19. The nose arrives at the abutment and the replacement of the temporary support.
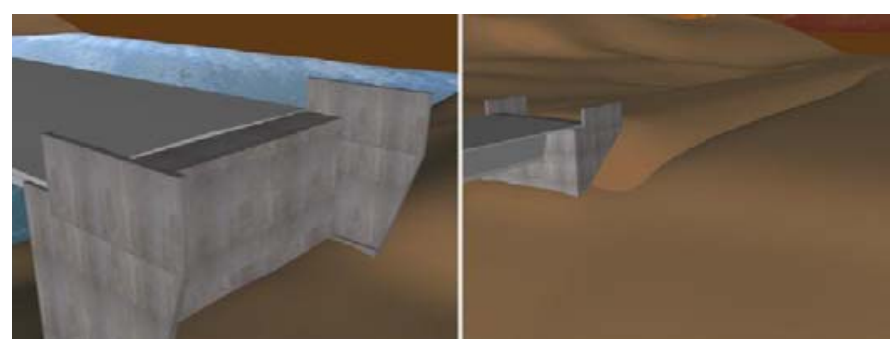

Fig. 20. The yard is removed and the space is covered.

The execution of virtual bridge could not end without provide the finishing which allow the sense of construction completed. For this operation, the camera moves along the bridge, while the guards and the finishing elements are positioned. So, finally the guards are positioned and also other finishing elements (Fig. 21).
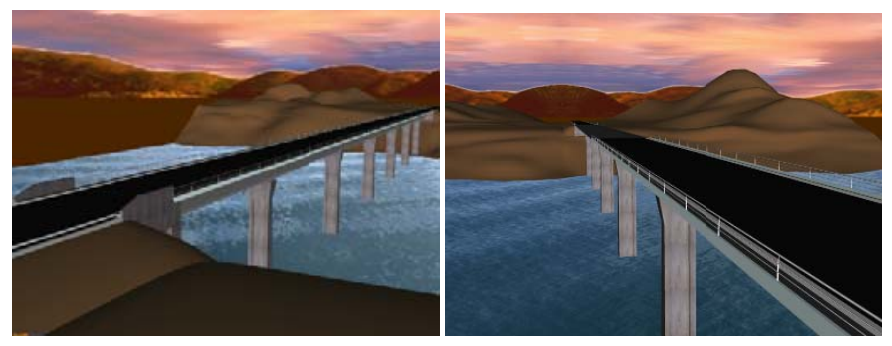

Fig. 21. The finishing elements are positioned over the deck.

The application resulted in a file with $4 \mathrm{mb} .113$ nodes were programmed manually resulting more than 2400 lines of code, 212 nodes of the type "keyframe", 122 nodes of the type "place", 15 nodes of the type "timesensor" among others. The model was created within a Master Thesis research work. 


\section{TRAINING ASPECTS}

The model was placed on a webpage thus being available for learners to manipulate. For that they should download the free EON Viewer application available at: http://download. eonreality.com

The bridge model particularly shows the complexity associated to the construction work of the deck. The model illustrates in detail the movement of the equipment. In class, the trainer must explain way the process must follow that sequence of steps and the way the equipment devices operates. When the learner goes to a real work place he can observe the complexity of the work and better understand the progression of construction previously explained [10].

This model presents a great complexity of geometry and material concerning the different elements used in a real work process. It provides an immersive capacity inherent to virtual world and it has a menu of events display allowing the learner to select a specific part he wants to observe again (Fig. 22).

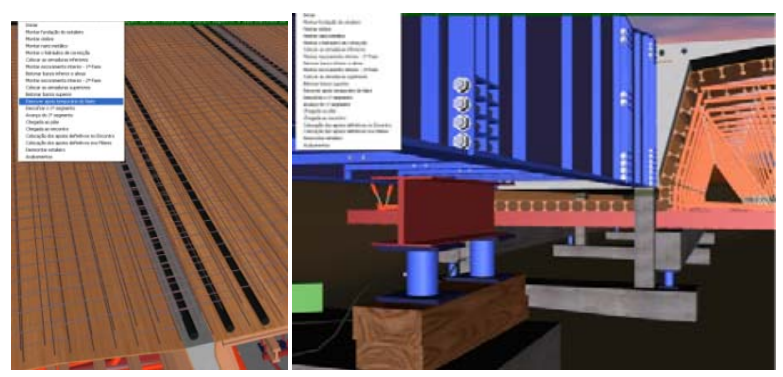

Fig. 22. Menu of events supports the interactivity with the model.

In the incremental lunching the camera movement shows the model in a consistent way to present all sequences of events allowing the user to perceive correctly the most important details of this construction method. During the animation, the position of the camera and its movement are synchronized to show the details of the elements or the assembly type and also an overview of the working place. The animation of the construction process can be visualized at: http://www.octaviomartins.com/lancamentoIncremental/

\section{SPREADING THE WORK}

In order to facilitate the use of the virtual model a film was made and it is available in: http://www.youtube.com/watch?v= S3Kf9e6JgF4\&feature=player_embedded

The film has recently support the description of the methodology of the incremental launching deck construction in a technical seminar session were several bridges construction processes were referrer. The detail of the configuration of each element involved in the performance, the camera positioned in an adequate way at each time where the construction activity is in progress and finally the accuracy of the construction sequence are good arguments to use the model or the film made over the virtual model as an education tool and as an important support to explain bridge construction technologies. At the real place it is quite difficult to show in detail all the work because visitors must be kept in a security distant from the bridge construction place. As documented in photos of Fig. 23 it is possible to evaluate how far the visitor has to be positioned in relation to the deck. The photos concern the construction of a bridge in Silleda, Spain .

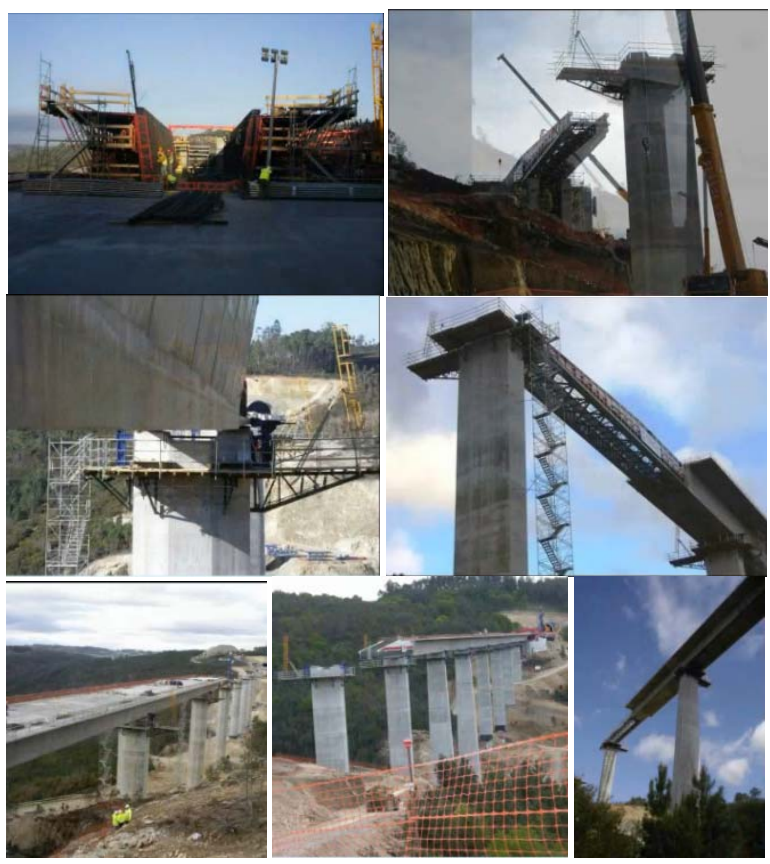

Fig. 23. Construction of Silleda's bridge, in Spain. http://www.youtube.com/watch?v=LKANQgf4LH8\&feature=player_embedd ed

At such a distance it is not possible to observe adequately every aspect. Comparing images included in Fig. 23 and Fig. 24 it is possible to recognize that the virtual model reaches every point of the construction and the student or the learner in a training technical session is able to better understand every detail of the process.

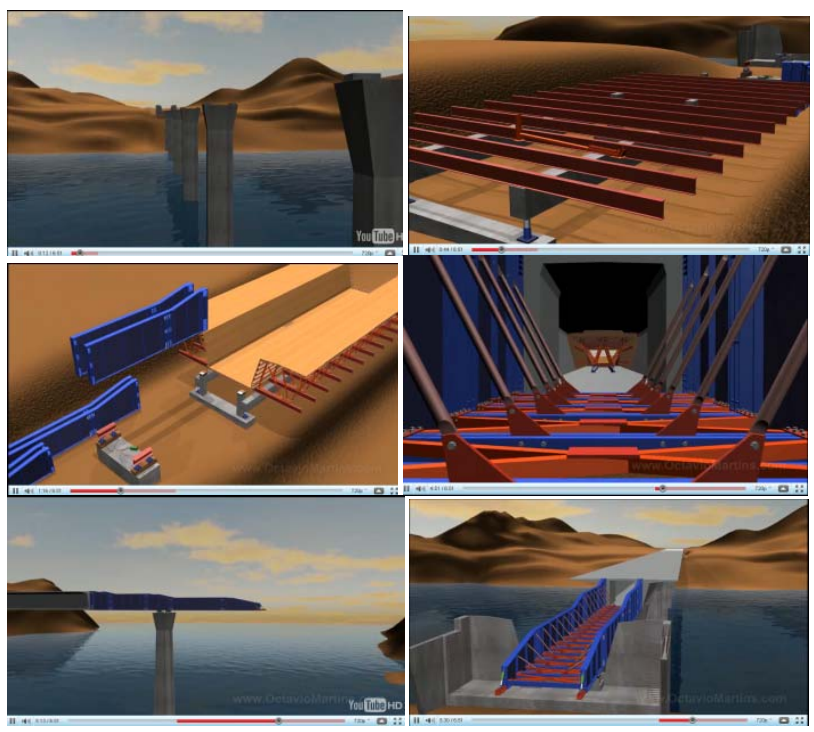

Fig. 24. Images from the film made over the virtual model. 


\section{CONCLUSIONS}

It has been demonstrated, through the example presented here, how the technology of virtual reality can be used in the elaboration of teaching material of educational interest in the area of construction processes.

The model generated represents the construction of a bridge in a standard situation. The student can interact with the virtual model in such a way that he can set in motion the construction sequence demanded by actual construction work, observe the methodology applied, analyse in detail every component of the work and the equipment needed to support the construction process and observe how the different pieces of a construction element mesh with each other and become incorporated into the model.

The model was placed in a repository created under the activities of e-school, a platform developed in the TU Lisbon, to be accessed by students and teachers of another institutions related to Civil Engineering. The application is oriented, not only as a learning tool, but also to professionals related to the construction of this kind of bridges. So, the model could be an important support to teachers to illustrate bridges construction issues in class and after, by themselves, using there PCs.

Compared with other visual representations, the application that resulted from this work, is: greater complexity of material and space in an attempt to provide more immersive capacity for its view; the introduction of a menu of events and displays, which allows the selection by the user of rapid events that he may want to view; concern with the user, trying to predict the possible sources of misunderstanding and giving them the prominence that it was considered necessary; showing the movements of the camera in a consistent way, to present all sequences of events not only in teaching but also in appealing to provide interest and attention from the user.

It is supposed that this work has the aim of providing not only the direct, fast and consistent understanding of the incremental launching method, but also show the capacity of representation in virtual environment in support of innovative techniques for construction.

\section{ACKNOWLEDGMENT}

The authors gratefully acknowledge the financial support of the Foundation for Science and Technology, a Governmental Organization for the research project PTDC/ ECM/67748/ 2006, now in progress.

\section{REFERENCES}

[1] A. Z. Sampaio, P. G. Henriques and C. O. Cruz. Visual simulation of construction activity supported on VR models: e-Learning tools. In: IADIS International Conference e-Society, Barcelona, Spain, pp. 284-291, 2009.

[2] O. P. Martins. Visual simulation of the incremental laughing method of bridges construction based on virtual reality technology, Bologna Master Thesis, TU Lisbon, IST, Lisbon, Portugal, 2009.

[3] A. Z. Sampaio, P. G. Henriques and C. O. Cruz. Visual Simulation on Engineering education: construction of a wall, a bridge and a roof. In:
ICE09, Intelligent Computing in Engineering, 16th International Workshop of EG-ICE, Berlin, Germany, pp. 250-257, 2009.

[4] A. J. Reis. Bridges. Didactic text. TU Lisbon, IST, Lisbon, Portugal, 2006.

[5] F. Leonhardt. Basic Principles of the Construction of Concrete Bridges, Volume 6. Interciência, RJ, 1982.

[6] J. Mathivat. Construction of bridges of concrete pre-stressed by successive advances. E.T.A., 1980.

[7] M. LaViolette. Bridge Construction Practices using Incremental Launching study. AASHTO, 2007.

[8] VSL. The Incremental Launching Method in Prestressed Concrete Bridge Construction, V.S.L. International Ltd, 1977.

[9] EON system, Introduction to working in EON Studio, EON Reality, Inc, 2008.

[10] O. P. Martins and A. Z. Sampaio. The incremental launching method for educational virtual model. In: CDVE2009, the $6^{\text {th }}$ International Conference on Cooperative Design, Visualization and Engineering, Luxembourg City, Luxembourg, Y. Luo (Ed.): CDVE 2009, LNCS 5738, 2009. (C) Springer-Verlag Berlin Heidelberg 2009, pp. 329-332, 2009.

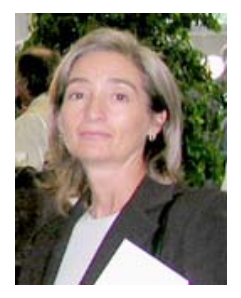

Alcínia Z. Sampaio, Assistant Professor at the Technical University of Lisbon (TULisbon), in the Department of Civil Engineering, $\mathrm{PhD}$ in Civil Engineering by TU Lisbon, since 1999, MSc in Structures Engineering by TU Lisbon, since 1989, and BSc in Civil Engineering by TU Lisbon, since 1983).

She teaches Technical Drawing since 1984 and Computer Added Drawing since 2003 for Civil Engineering students. She has organized short courses in Nov. 2010, Introduction to Virtual Reality Technology applied on architecture and Engineering, TU Lisbon and in Feb. 2009, Automatic generation of drawing using DXF files, TU Lisbon.

The main research fields are Geometric modeling and virtual reality applied to construction. She has supervised the researcher project Automatically generating model of bridges graphic representation, (2001-2004) and has collaborated with the projects Virtual reality in optimisation of construction project planning, (2001-2004). Actually she is the principal researcher of the project Virtual Reality Technology applied as a support tool to the planning of construction maintenance, (2008-2010). 